Each ring was suspended from the ceiling with a cord, its major axis being kept vertical. To its lower end a weight of $4.5 \mathrm{~kg}$. was applied. This caused an appreciable lateral contraction of the ring along the minor axis amounting to a few $\mathrm{mm}$.

The ring was then pulled out laterally along the minor axis until it resumed its initial length before the loading, while the corresponding force was measured by a spring balance.

The results of the calculations and the experiments are summarized below:

\begin{tabular}{|c|c|c|c|c|c|c|c|}
\hline \multirow{3}{*}{$\begin{array}{l}\text { Circle } \\
(20 \mathrm{~cm} . \text { dia. }) \\
\text { Ellipse }\end{array}$} & & \multicolumn{3}{|c|}{ Calculated } & \multicolumn{3}{|c|}{ Experimeated } \\
\hline & $a_{1}$ & $a_{2}$ & $B$ & 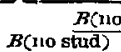 & $\frac{\text { o stud }}{\boldsymbol{B}}$ & & \\
\hline & -1.90 & 0.5 & 0.30 & 0.64 & 2.1 & 1 & 1 \\
\hline $\begin{array}{l}\text { Enpse } \\
(20 \mathrm{~cm} . \times 30 \mathrm{~cm} .) \\
\text { Ellipse }\end{array}$ & -1.16 & 0.2 & 0.28 & 0.51 & 1.8 & 0.64 & 0.62 \\
\hline$(20 \mathrm{~cm} \cdot \times 40 \mathrm{~cm})$. & -0.8 & 0.3 & 0.28 & 0.41 & 1.5 & 0.38 & 0.40 \\
\hline
\end{tabular}

Considering the approximate nature of our calculation and allowing for irregularities of elastic property which may exist in the model despite every effort done to avoid them, the agreement between the calculated and experimental results is quite satisfactory.

By means of the foregoing research,' we may find the scantling of the ring of uniform strength for a studded chain. For practical purposes, its wear and, tear being duly taken into consideration, suitable scantling may be assigned. This provides a chain of greater length for equal weight or a lighter chain for equal length when compared with that generally and universally used at the present time.

The above model experiment was made with an allowance from the Minister for Education, out of our government appropriation for the encouragement of scientific researches for the fiscal year 1951, for which the author offers his. grateful thanks.

It is desirable to push the experiments further with actual chains instead of simple models and to finish up with their breaking tests, in order that the practical application of the present research may bear direct and beneficial fruits for the industry in general. (End)

\title{
On Vibration of a Cylindrical Shell, which is Filled with Water.*
}

\author{
By Fumiki Kıтo**
}

\begin{abstract}
The Author has made hydrodynamical study on vibratory motion of a cylindrical shell, in which water is filled, and has obtained the amount of virtual mass of water for the case where both ends of the cylinder are fitted with rigid end-plates.
\end{abstract}

\section{Introduction}

When a cylindrical shell, inside which water is filled, makes a vibratory motion, the water contained inside the shell also makes vibratory motion. The effect of this vibratory motion of water is to increase the apparent mass of the shell and lower its natural frequency of vibration as compared to values when there is no water. This effect is called the virtual mass of water. In the present paper, the Author gives some results of theoretical estimations of this- virtual mass for the case of a vibration of a shell in form of a circular cylinder, both ends of which are left free, or are closed with rigid end-plates.

According to the calculation, the effect of rigid end plates appear as a kind of "end effect", which has significant influence only if the ratio of the length of cylindrical shell to its radius is less than 5 .

2. Solution for the case of a series of cylinders.

Let us consider a cylindrical shell of radius $a$

* Read befóre Meetings on Vibration and Wave-Motion.

Mar. 15, 1953. Received Sept. 28, 1953

** Department of Engineeting, Keio University. 
and length $l$, as shown in Fig. 1. We take $x$ axis along the center line of the shell.

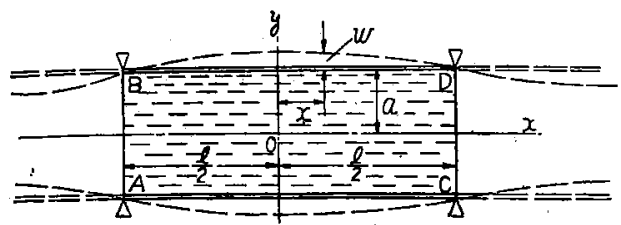

Fig. 1. Cylindrical Shell.

In this section, we treat the case in which there exist a series of cylinders of same dimensions along the $x$-axis, Fig. 1 showing one of the series.

The shell is supposed to be supported at each ends, that is at $x= \pm l / 2$, and make vibration with frequency $f$. The angular frequency corresponding to it is $\omega=2 \pi f$.

When the water is filled in the shell, the water will also make vibratory motion, with the same angular frequeney $\omega$. If the travelling velocity of pressure (or sound) wave in water is $C$, the wave length is given by $\lambda=c / f$. For example, if $c=1460 \mathrm{~m} / \mathrm{sec}$. and $f=50 / \mathrm{sec}$., then $\lambda=1460 \div$ $50=29.2 \mathrm{~m}$. Usually the dimensions $2 a$ or $l$ of the shell is small in comparison with the. wave $\operatorname{len}_{\alpha}$ th $\lambda$.

Now, the vibratory motion of the water is to be determined from the equation of wave motion;-

$$
\frac{\partial^{2} \phi}{\partial x^{2}}+\frac{\partial^{2} \phi}{\partial y^{2}}+\frac{\partial^{2} \phi}{\partial z^{2}}=\frac{1 \cdot \partial^{2} \phi}{c^{2}} \frac{\partial t^{2}}{\partial t^{2}}
$$

But, if the wave length $\lambda$ is very large in comparison to the main dimensions of the shell, we may use the equation

$$
\frac{\partial^{2} \dot{\phi}}{\partial x^{2}}+\frac{\partial^{2} \phi}{\partial y^{2}}+\frac{\partial^{2} \phi}{\partial z^{2}}=0, \text { or } \nabla^{2} \phi=0
$$

instead of the above equation (1), which corresponds to neglecting the compressibility of water. $\phi$ is the velocity potential.

A solution of equation (2) in terms of cylindrical co-ordinates $(r, \theta, x)$ may be written

$$
\phi_{1}=A \cos k x \sin n \theta \ln (k r) \sin \omega t
$$

Corresponding to this value of $\phi_{1}$ we have

$$
\left.\begin{array}{l}
V_{r}=\frac{\partial \phi_{1}}{\partial r}=k A \cos k x \sin n \theta I_{r^{\prime}}(k r) \sin \omega t \\
V_{x}=\frac{\partial \phi_{1}}{\partial x}=-k A \sin k k_{1} \sin n \theta I_{n}(k r) \sin \omega t
\end{array}\right\}
$$

Now, let us assume that the wall of the shell is making vibration, its radial displacement $w$ being given by

$$
w=W \cos k x \sin n \theta \cos \omega t
$$

where $W$ is a constant. If the water is vibrating together with the shell, we must have at $r=a, V_{r}=\partial w / \partial t$. Whence we have by (4):-

$$
A=-\frac{\omega W}{k I_{n^{\prime}}\left(k r_{0}\right)}
$$

In what follows, we shall take up the case in which $k=\pi / l$, but by slight change of expression, we can estimate the case in which $k=m \pi / l$, where $m$ is an odd integer.

The kinetic energy at any instant of water contained in one span $(x=-l / 2$ to $+l / 2)$ of the cylinder is given by

$$
T_{w}=-\rho_{w} \int_{\theta=0}^{2 \pi} a d \theta \int_{x=\theta}^{2 \pi / k}\left[\phi \frac{\partial w}{\partial t}\right] d x
$$

where $\rho_{w}$ is the density of water.

Or, putting the value of $\phi_{1}$ as given by (3):-

$$
T_{w}=\rho_{w} \frac{\omega^{2} a}{k} W^{2} \frac{I_{n}(k a)}{I_{n}^{\prime}(k a)} \frac{\pi^{2}}{k} \sin ^{2} a^{\prime} t
$$

While the amount of kinetic energy of wall of shell, whose thickness is $2 h$ and density $\rho_{m}$, is given by

$$
\begin{aligned}
T_{m} & =\rho_{m} \int_{\theta=0}^{2 \pi} a d \theta \int_{x=0}^{2 \pi / k}\left(\frac{\partial w}{\partial t}\right)^{2} \cdot \frac{2 h}{2} \cdot d x \\
& =\rho_{m} \omega^{2} W^{2} a h \frac{\pi^{2}}{k} \sin ^{2} \omega t .
\end{aligned}
$$

And the ratio $T_{w} / T_{m}$ is

$$
\varepsilon=\frac{T_{w}}{T_{m}}=\left(\frac{\rho_{w}}{\rho_{m}}\right)\left(\frac{a}{h}\right) \frac{I_{n}(K)}{n I_{n}(K)+K I_{n+1}(K)}
$$

where we have put $K=k a=\pi a / l$. If the water exist outside of the shell (instead of inside) and extend to infinity, while the inside of the shell is vacant, we should have, instead of (3),

$$
\phi_{1}=A \cos k x \sin n \theta K_{n}(k r) \sin \omega t
$$

and, by making the similar calculations, we obtain

$$
\varepsilon^{\prime}=\frac{T_{w}}{T_{m}}=\left(\frac{\rho_{w}}{\rho_{m}}\right)\left(\frac{a}{h}\right)\left[\frac{-K_{n}(K)}{n K_{n}(K)-K K_{n+1}(K)}\right]
$$

If the water exist both inside and outside of the shell, we must take the sum $\varepsilon+\varepsilon^{\prime}$ of the above two values in order to obtain the ratio $T_{w} / T_{m}$. In Table $I$, some values of the coefficients

$$
\begin{aligned}
& M_{1}=\frac{I_{2}(K)}{n I_{n}(K)+K I_{n+1}(K)} \\
& M_{1}^{\prime}=\frac{-K_{n}(K)}{n K_{n}(K)-K^{\prime} K K_{n+1}(K)}
\end{aligned}
$$


are shown.

Table 1. Values of $M_{1}$ and $M_{1}^{\prime}$

\begin{tabular}{|c|c|c|c|c|c|}
\hline & & $K=0$ & $K=0.2$ & $K=0.4$ & $K=0.6$ \\
\hline$M_{1}$ & $\begin{array}{r}n=2 \\
3 \\
4\end{array}$ & $\begin{array}{l}0.5000 \\
0.3333 \\
0.2500\end{array}$ & $\begin{array}{l}0.4993 \\
0.3328 \\
0.2498\end{array}$ & $\begin{array}{l}0.4935 \\
0.3311 \\
0.2490\end{array}$ & $\begin{array}{l}0.4566 \\
0.3284 \\
0.2478\end{array}$ \\
\hline$M_{1}^{\prime}$ & $\begin{array}{r}n=2 \\
3 \\
4 \\
4\end{array}$ & $\begin{array}{l}0.5000 \\
0.3333 \\
0.2500\end{array}$ & $\begin{array}{l}0.4952 \\
0.3332 \\
0.2496\end{array}$ & $\begin{array}{l}0.4865 \\
0.3286 \\
0.2483\end{array}$ & $\begin{array}{l}0.4645 \\
0.3242 \\
0.2464\end{array}$ \\
\hline
\end{tabular}

3. Solution for the case of cylindrical shell with rigid bottom plates.

With the above mentioned value of $\phi_{1}$, the axial velocity $V_{x}$ of water at ends $x= \pm l / 2$ have some values and are not identically zero. If we wish to obtain the solution for the case of rigid end-plates, we must add to $\phi_{1}$ another potential $\phi_{2}$ such that,

(a) $\nabla^{2} \phi_{2}=0$, throughout the inside of the shell

(b) at $r=a, \frac{\partial \phi_{2}}{\partial r}=0$

(c) at $x= \pm \frac{l}{2}, \quad \frac{\partial \phi_{1}}{\partial x}+\frac{\partial \phi_{2}}{\partial x}=0$.

We take

$\phi_{2}=\sum_{i} B_{i} \cosh m_{i} x \sin n \theta J_{n}\left(m_{i} r\right) \sin \omega t$

which satisfy the equation $\nabla^{2} \phi_{2}=0$. According to the condition (b), we must have

$$
J_{n^{\prime}}\left(m_{i} a\right)=0
$$

According to the condition (c), we have, at $x= \pm l / 2$,

$-A k \sin k x \sin n \theta I_{n}(k r)$

$+b \sum_{i} B_{\imath} m_{\imath} \sinh m_{\imath} x \sin n \theta J_{n}\left(m_{\imath} r\right)=0$

If we put for shortness,

$$
B_{\imath} m_{\imath} \sinh m_{\tau} \frac{l}{2}=A \frac{\pi}{l}-C_{\imath}
$$

the above equation reduces to:-

$$
I_{n}\left(\pi \frac{r}{l}\right)=\sum_{i} C_{t} J_{n}\left(m_{l} r\right) \quad 0 \leqq r \leqq a
$$

which means that, when we expand the function at the left hand side of this equation as a series of Bessel functions $J_{n}\left(m_{t} r\right)$, the coefficients of expansion are $C_{\imath}$.

Now, according to the known formula)

$$
\begin{gathered}
\int_{a}^{n} J_{i n}\left(m_{\xi} r\right) J_{n}\left(m_{j} r\right) r d r=0 \quad(j \neq i) \\
\int_{0}^{a}\left[J_{n}\left(m_{\imath} r\right)\right]^{2} r d r=\frac{1}{2 m_{\imath}{ }^{2}}\left[\left(m_{\imath}{ }^{2} a^{2}-n^{2}\right) \overline{J_{n}\left(m_{\imath} a\right)^{2}}\right]
\end{gathered}
$$

the coefficients $C_{\imath^{\prime}}$ can be easily determined thus;-

$$
\begin{aligned}
& \frac{C_{8}{ }^{2}}{2 m_{\imath}{ }^{2}}\left[\left(m_{\imath}{ }^{2} a^{2}-n^{\prime}\right) \overline{J_{n}\left(m_{\imath} a\right)^{2}}\right] \\
&=\int_{0}^{a} I_{n}\left((k r) J n\left(m_{i} r\right) r d r\right. \\
&=\frac{a}{k^{2}+m_{\imath}{ }^{2}}\left[k J_{n}\left(m_{\imath} a\right) I_{n^{\prime}}(k a)\right. \\
&\left.-m_{\imath} I_{n}(k a) J_{n}^{\prime}\left(m_{\imath} a\right)\right]^{2)} \\
&= \frac{a}{k^{2}+m_{8}{ }^{2}}\left[k J_{n}\left(m_{\imath} a\right) I_{n}(k a)\right]
\end{aligned}
$$

Knowing thus the value of constants $C_{q}$, the values of $B_{\imath}$ can be calculated, and we have

$$
\begin{aligned}
B_{\imath}= & A \frac{2 m_{\imath} k^{2} a}{\sinh \left(\frac{1}{2} m_{\imath} l\right)} \cdot \frac{1}{\left(k^{2}+m_{\imath}^{2}\right)\left(m_{i}^{2} a-n^{2}\right)} \\
& \times \frac{I_{n^{\prime}}(k a)}{J_{n}\left(m_{\imath} a\right)}
\end{aligned}
$$

The value of kinetic energy of water contained in the region $x= \pm l / 2$ is,

$$
T_{w}=-\rho_{w} \int_{\theta=0}^{2 \pi} a d \theta \int_{x=-l / 2}^{x=+l / 2}\left[\left(\phi_{1}+\phi_{2}\right) \frac{\partial w}{\partial t}\right] d x
$$

Putting the values of $\phi_{1}$ and $\phi_{2}$ into this expression, and utilizing the formula

$$
\begin{aligned}
& \int_{-3 / 2}^{+l / 2} \cos k x \cosh m_{\imath} x d x \\
& \quad=\frac{1}{2}\left|\frac{\exp \left(m_{\imath} x\right)\left[m_{\imath} \cos k x+k \sin k x\right]}{\left(k^{2}+m_{\imath}^{2}\right)}\right| \\
& \quad=\frac{2 k}{k^{2}+m_{t}^{2}} \cosh \left(\frac{1}{2} m_{\imath} l\right)
\end{aligned}
$$

we have at last

$$
\begin{aligned}
T_{w} & =\rho_{w} a \frac{W^{2}}{k} \frac{I_{n}(k a)}{I_{n}(k a)} \omega^{2} \pi . \\
& \times\left[\frac{l}{z}+\sum_{i} \frac{B_{l}}{A} \frac{J_{n}\left(m_{q} a\right)}{I_{n}(k a)} \cdot \frac{2 k \cosh \left(\frac{1}{q} m_{l} l\right)}{k^{2}+m_{l}^{2}}\right]
\end{aligned}
$$

Comparing this value with the expression for $T_{m}$ as obtained in the previous section, we have,

$$
\begin{aligned}
\varepsilon & =\frac{T_{w}}{T_{m}}=\left(\frac{\rho_{w}}{\rho_{m}}\right)\left(\frac{a}{h}\right) \frac{I_{n}(k a)}{I_{n}(k a) \cdot k a} \\
& \times\left[1+\sum_{i} \frac{B_{l}}{A} \frac{m_{\imath} a}{I_{n}(k a)} \cdot \frac{2 k^{2} \cosh \left(\frac{1}{2} m_{z} l\right)}{\left(k^{2}+m_{l}{ }^{2}\right)}\right]
\end{aligned}
$$

This expression can be transformed to a more convenient form, as follows; -

$$
\varepsilon=\frac{\rho_{w}}{\rho_{m}} \cdot \frac{a}{h} \cdot M_{2}
$$

where we have

$$
M_{2}=M_{1}+\sum_{i} \frac{\left(\frac{4 l}{\pi a}\right) \xi_{i}}{\left[1+\left(\xi_{i} \frac{l}{\pi a}\right)^{2}\right]^{2}} \cdot \frac{\operatorname{coth} \frac{\xi_{l} l}{2 a}}{\left(\xi_{t}^{2}-n^{2}\right)}
$$

where $M_{1}$ is the value obtained in the previous 
section. $\xi_{1}$ are the roots of the equation

$$
J_{n}(\xi)=0
$$

and have following values:--

$$
\begin{array}{llll}
n=2 & \xi_{1}=3.05 & \xi_{2}=6.7 & \xi_{3}=7.9 \\
n=3 & \xi_{1}=4.3 & \xi_{2}=8.0 & \xi_{3}=11.4 \\
n=4 & \xi_{1}=5.3 & \xi_{2}=9.3 &
\end{array}
$$

The values of $M_{1}$ and $M_{2}$ for a range of values of $l / a$ up to 5 are shown in Fig. 2. From the figare we see that for $l / a>5$, we may take $n M_{1}$, $n M_{2}$ to be approximately equal to unity.

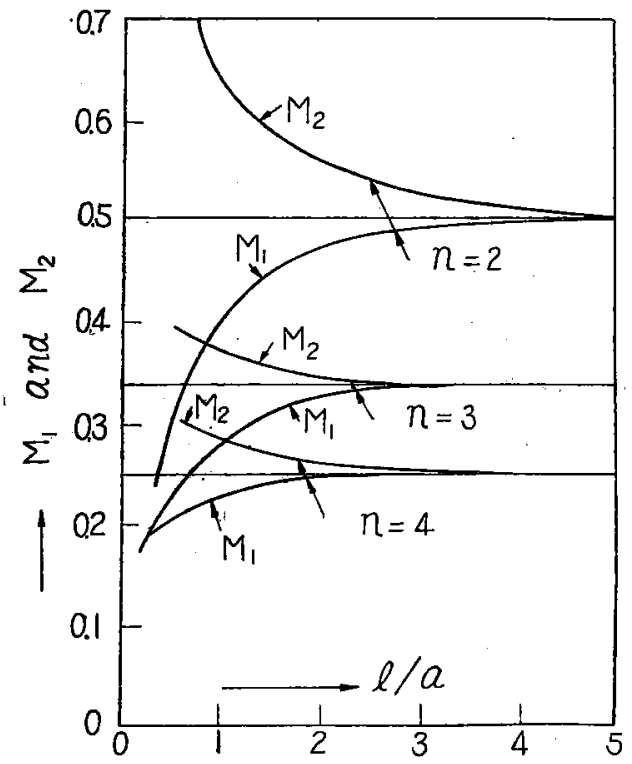

Fig. 2. Values of $M_{1}$ and $M_{2}$

\section{A Remark on other cases.}

The Author has also made a discussion about the same problem for various arrangements of cylindrical shells as shown in Fig. 3 .

In this Figure the thick lines show rigid pipe walls, while the slender lines represent pipe walls making vibration. Fuller account about these cases will be reported in a forthcoming issue of the Proc. of Faculty of Engineering, Keio University.

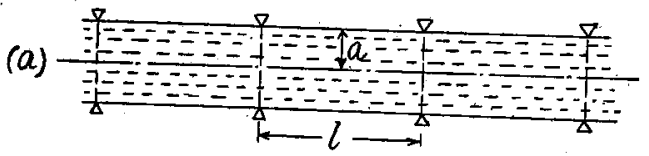

(b)

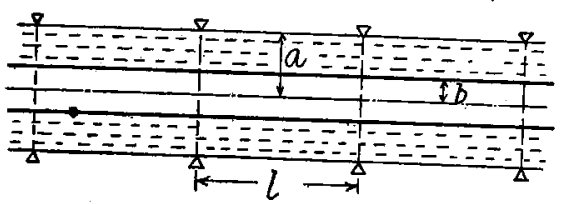

(c)

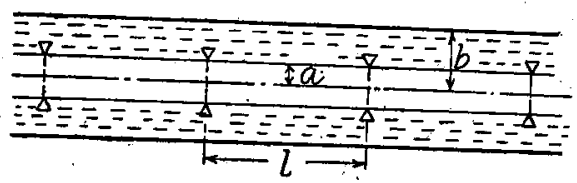

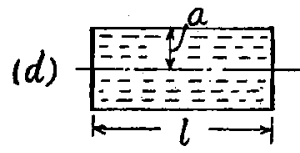

(e)

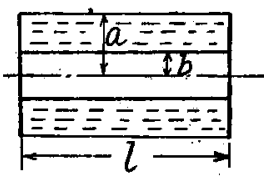

(f)

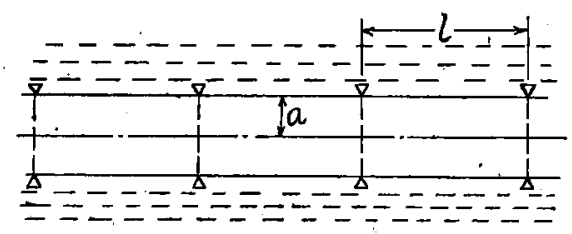

(g)

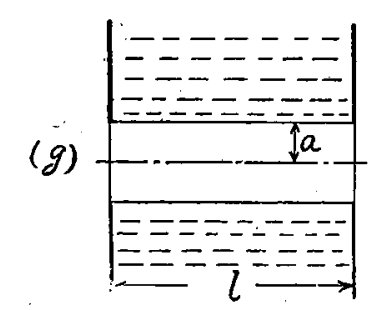

(h)
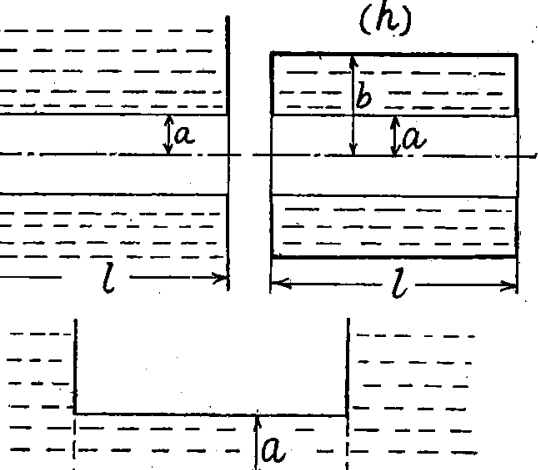

(i) $=-1=-2=-1+1-\ldots$

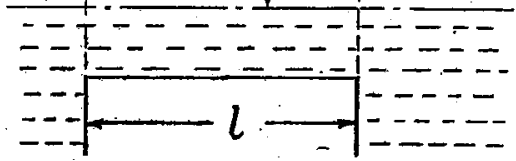

Fig. 3. Various arrangements of cylindrical shells.

\section{References}

1) H. Lamb, Hydrodynamics, \& 191.

2) MacLachlan. Bessel Functions for Engineers, p. 115. 